\title{
Preliminary Study for the Determination of Rare Earth Elements Using the ICP-MS Technique
}

\author{
GINA ALINA CATRINA (TRAISTARU) ${ }^{1}$, GABRIELA GEANINA VASILE ${ }^{1 *}$, \\ IONUT NICOLAE CRISTEA ${ }^{1}$, GEORGIANA CERNICA ${ }^{1}$, CRISTINA ILEANA COVALIU ${ }^{2}$, \\ LUOANA FLORENTINA PASCU ${ }^{1}$ \\ ${ }^{1}$ National Research and Development Institute for Industrial Ecology - ECOIND, 71-73 Drumul Podu Dambovitei Str., \\ 060652 Bucharest, Romania \\ ${ }^{2}$ University Politehnica of Bucharest, Faculty of Biotechnical Systems Engineering, 313 Splaiul Independentei Str., \\ 060042, Bucharest, Romania
}

\begin{abstract}
This paper proposes an optimized method for the determination of rare earth elements (Sc, $Y, \mathrm{La}, \mathrm{Ce}, \mathrm{Pr}, \mathrm{Nd}, \mathrm{Sm}, \mathrm{Eu}, \mathrm{Gd}, \mathrm{Tb}, \mathrm{Dy}, \mathrm{Ho}, \mathrm{Er}, \mathrm{Tm}, \mathrm{Yb}, \mathrm{Lu})$ from soils using ICP-MS technique. First, the soil samples were thermal treated at three different temperatures $550^{\circ} \mathrm{C}, 700^{\circ} \mathrm{C}$ and $850^{\circ} \mathrm{C}$ in order to eliminate organic matter interferences. Then, the residual samples remaining from the calcination process were extracted in acidic medium with two different digestion methods (method I - a mixture of nitric acid and hydrogen peroxide; method II - aqua regia mixture) in order to quantify rare earth elements content. The highest recovery percentages for the major rare earth elements analyzed (Sc, $Y$, $\mathrm{La}, \mathrm{Ce}, \mathrm{Pr}, \mathrm{Nd}$. Sm) were situated in the range $86.13 \%$ to $99.90 \%$, in sample residues thermally treated at $700^{\circ} \mathrm{C}$ and extracted with nitric acid and hydrogen peroxide.
\end{abstract}

Keywords: ICP-MS, optimized method, rare earth metals, soil

\section{Introduction}

The rare earth elements (REEs) represent a group of 17 elements, which contain 15 lanthanides (lanthanum $(\mathrm{La})$, cerium $(\mathrm{Ce})$, praseodymium $(\mathrm{Pr})$, neodymium $(\mathrm{Nd})$, promethium $(\mathrm{Pm})$, samarium $(\mathrm{Sm})$, europium $(\mathrm{Eu})$, gadolinium $(\mathrm{Gd})$, terbium $(\mathrm{Tb})$, dysprosium (Dy), holmium (Ho), erbium (Er), thulium (Tm), ytterbium (Yb), lutetium (Lu)), scandium (Sc) and yttrium (Y). The lanthanides are very important in green-economy, because in modern technology these elements are used in different devices: permanent magnets, lamp phosphors, rechargeable nickel metal hydride batteries, catalysts, medical-devices and also for other application $[1-9,10]$.

Lanthanides are present in lower concentration in the composition of different types of soils: limestone, magmatic, clay and sandy soils, therefore advanced and sensitive analytical methods are required [10-15].

The European Commission and U.S. Department of Energy mention the lanthanides as the most critical raw materials for all the industries, five critical rare earths being europium, terbium, dysprosium, neodymium and yttrium $[15,16]$.

Recovering and separating REEs from mineral sources constitutes the first step for their commercial applications in technical, medical and agricultural areas. The REEs are used in high-tech industry, electronics and medicine, so it was stimulated the geochemists interest on their environmental behaviour and potential toxicity $[17,18]$.

The most important anthropic sources of metals in the environment are industry (metallurgy, chemistry, construction and mining), incinerators of urban or polluted residues, auto traffic, agriculture as well as domestic activities [18-20]. Different natural or artificial sources of pollution can pollute the soil and sediments with rare earths and metals. In nature, REEs become available to the soil, entering in groundwater body, and consequently to plants, by leaching from mineral deposits or due to use of

*email: gabriela.vasile@incdecoind.ro 
phosphate fertilizer. The presence of rare earth elements and metals in soil can affect the quality of food, groundwater, microorganism's activity, plant growth $[19,20]$.

Detection and quantification of metals in soil is usually performed with two sensitive and selective techniques, such as inductively coupled plasma optical emission spectrometry (ICP-OES) and inductively coupled plasma mass spectrometry (ICP-MS). At ultra-trace levels, due to its higher sensitivity, ICP-MS technique is preferred [20-24].

The aim of the study was to establish an optimized procedure for soil pretreatment in order to quantify REEs content at the highest recovery rate, determination of REEs concentration being performed with ICP-MS technique. Thermogravimetric analysis were performed on soil samples in order to establish organic matter content, possible interference in REEs determination. The proposed procedure was applied on agricultural soil samples with clay structure.

\section{Materials and methods}

Six soil samples were collected from Hunedoara County (P1, P2, P3) and Sibiu County (P4, P5, P6). The sampling points are represented in Figure 1, soil samples with clay structure were collected according to international standards, from $30 \mathrm{~cm}$ depth.

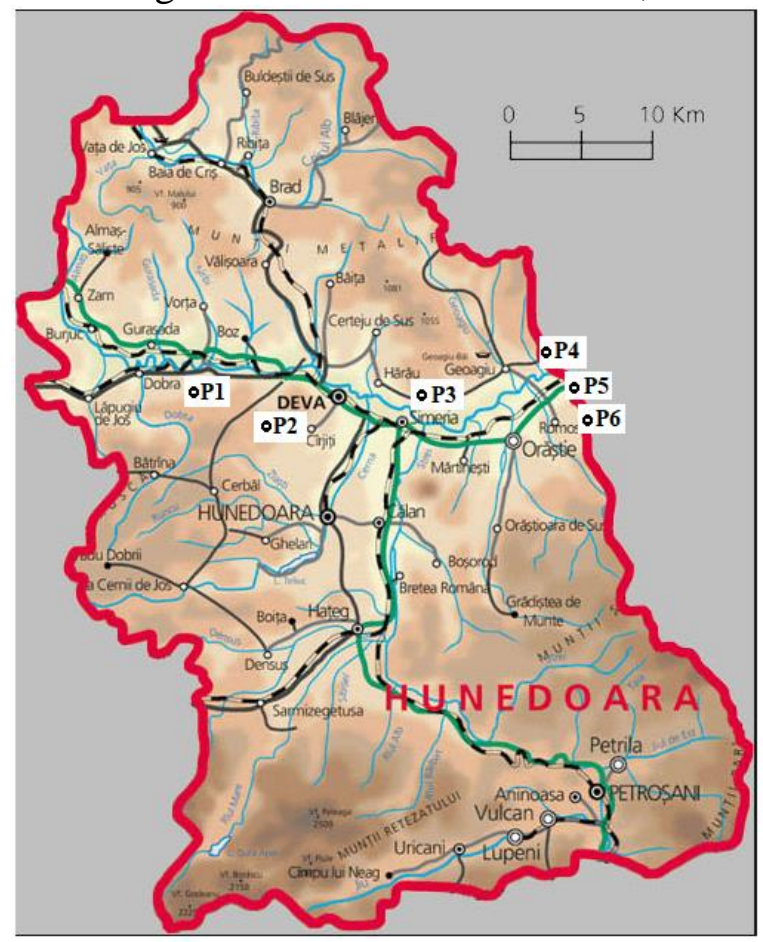

Figure 1. Locations of soil sampling points

The geographic coordinates, description of the sampling points as well as the sample codes are shown in Table 1.

Table 1. Geographic coordinates of sampling points

\begin{tabular}{cccc}
\hline No. & $\begin{array}{c}\text { Sample } \\
\text { code }\end{array}$ & Sampling locations & GPS coordinates \\
\hline \multirow{2}{*}{1} & P1 & Dobra, 1 Decembrie Street, Hunedoara & 45.907950 \\
& & County & 22.581600 \\
\hline \multirow{2}{*}{2} & \multirow{2}{*}{ P2 } & Deva, Santuhalm Street, Hunedoara & 45.857440 \\
& & County & 22.945810 \\
\hline \multirow{3}{*}{3} & \multirow{4}{*}{ P3 } & Simeria \\
& & DN 7, km 337+380, & 45.834960 \\
& \multirow{4}{*}{4} & Hunedoara County & 23.035800 \\
& P4 & Sibiu County \\
& & Km 296+300 - DN1 & 45.699900 \\
& & Km 258+790 - DN7 & 24.249700 \\
\hline
\end{tabular}




\begin{tabular}{cccc}
\hline \multirow{5}{*}{5} & Sibiu County \\
& P5 & A1 highway Sebes-Sibiu, & 45.782680 \\
& & Km76 + 150 & 24.011400 \\
\hline 6 & P6 & Sibiu County & 45.789450 \\
& & Alba Iulia Street & 24.085920 \\
\hline
\end{tabular}

\section{Sample preparation}

The soil samples were dried at room temperature and sieved. The fraction with particle dimension less than $150 \mu \mathrm{m}$ was retained and homogenized prior to analysis.

In order to concentrate the REEs content in soil samples, a thermal treatment was applied at $550^{\circ} \mathrm{C}$, $700^{\circ} \mathrm{C}$, respectively $850^{\circ} \mathrm{C}$ for $2 \mathrm{~h}$ in a calcination oven $[24,25]$.

In Figure 2 is presented the flow treatment of soil samples.

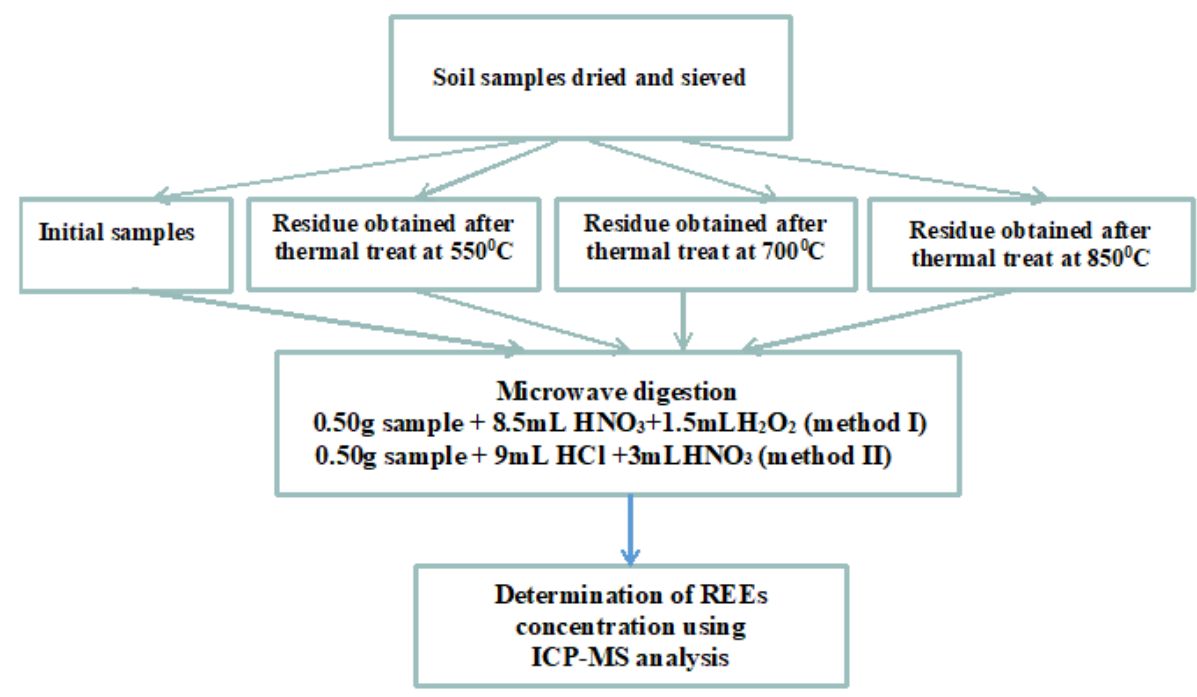

Figure 2. Flow treatment of soil samples

The residues of the samples, obtained after calcination at different temperatures, were digested using the program presented in Table 2. The same program was applied also to the "initial sample" (fig. 2) in order to analyze $\mathrm{As}, \mathrm{Cd}, \mathrm{Cr}, \mathrm{Mn}, \mathrm{Ni}, \mathrm{Pb}$ and $\mathrm{Zn}$.

Table 2. Digestion program

\begin{tabular}{ccccc}
\hline Step & $\mathbf{T 1}^{\mathbf{0}} \mathbf{C}$ & $\mathbf{T 2}^{\mathbf{0}} \mathbf{C}$ & Power $(\mathbf{W})$ & Time (min) \\
\hline 1 & 140 & 100 & 1600 & 30 \\
\hline 2 & 140 & 100 & 1600 & 35 \\
\hline 3 & cooled & cooled & - & 30 \\
\hline
\end{tabular}

After digestion, the solutions were filtered through a $0.45 \mu \mathrm{m}$ membrane and brought to $25 \mathrm{~mL}$ with ultrapure water. Subsequently, the metal concentrations were determined with an ICP-MS equipment.

\section{Materials}

All the reagents were of analytical quality grade. Volumetric flask and plastic recipients used for sampling and sample treatments were cleaned with $10 \% \mathrm{HNO}_{3}$ ultrapure and then washed with ultrapure water.

10 mg/L ICP-MS Multielement standard solution (Sc, Y, La, Ce, Pr, Nd, Sm, Eu, Gd, Tb, Dy, Ho, Er, Tm, Yb, Lu), Sigma Aldrich quality, was used for calibration curves.

Calibration curves for $\mathrm{As}, \mathrm{Cd}, \mathrm{Cr}, \mathrm{Mn}, \mathrm{Ni}, \mathrm{Pb}, \mathrm{Zn}$ were performed using $10 \mathrm{mg} / \mathrm{L}$ Multielement Certified Reference Material (ICP multi-element standard solution XXI, Certipur, Merck). Quality 
control of the analytical results for each element (REEs, other metals) was performed using monoelement Certified Reference Materials, $1000 \mathrm{mg} / \mathrm{L}$, Sigma Aldrich quality.

ERM-CC141 Loam Soil Multi-element Certified Reference Material, soil produced by Institute for Reference Materials and Measurements JRC Belgium, was used for quality control of metals (As, Cd, $\mathrm{Cr}, \mathrm{Mn}, \mathrm{Ni}, \mathrm{Pb}, \mathrm{Zn}$ ) concentrations in soil matrix.

\section{Equipment}

Microwave Digestion System Ethos Up Milestone; ICP-MS type 7900 Agilent with Mass Hunter 4.4 software; Thermogravimetric analyzer TG Netzsch STA 409; Millipore Milli-Q Ultrapure Water System; Calcination oven type Memmert UF 110.

\section{Metal analysis}

The ICP-MS operating parameters used for determination of REEs (Sc, Y, La, Ce, Pr, Nd, Sm, Eu, $\mathrm{Gd}, \mathrm{Tb}, \mathrm{Dy}, \mathrm{Ho}, \mathrm{Er}, \mathrm{Tm}, \mathrm{Yb}, \mathrm{Lu}$ ) and other metals (As, Cd, Cr, Mn, Ni, Pb, $\mathrm{Zn}$ ) are presented in Table 3. A collision cell was used to eliminate interference from chemical reagents and sample composition.

Table 3. Spectrometer operating parameters

\begin{tabular}{|c|c|}
\hline \multicolumn{2}{|c|}{ ICP-MS spectrometer parameters } \\
\hline Delay time: $60 \mathrm{~s}$ & Purge gas flow: normal \\
\hline Replicates: 3 times & Peristaltic pump: $1.5 \mathrm{~mL} / \mathrm{min}$ \\
\hline \multicolumn{2}{|c|}{ Tune parameters } \\
\hline \multicolumn{2}{|c|}{ Plasma parameters } \\
\hline Plasma flow rate: $15 \mathrm{~L} / \mathrm{min}$ & Power RF: $1550 \mathrm{~W}$ \\
\hline Auxiliary flow rate:0,90L/min & Plasma view: axial \\
\hline Nebulizer Pump: 0.10 rps & RF matching $1.30 \mathrm{~V}$ \\
\hline \multicolumn{2}{|c|}{ Plasma mode } \\
\hline Plasma Mode: General Purpose & Sample Depth: $10 \mathrm{~mm}$ \\
\hline \multicolumn{2}{|c|}{ Cell parameters } \\
\hline He Flow: $4.1 \mathrm{~mL} / \mathrm{min}$ & OctpBias: $-8.0 \mathrm{~V}$ \\
\hline \multicolumn{2}{|c|}{ Spectral peak processing } \\
\hline Peak algorithm: Peak area & Peak pattern: 3 points \\
\hline Replicates: 3 & Integration time: $0.6001 \mathrm{sec}$. \\
\hline \multicolumn{2}{|c|}{ Sample acquisition } \\
\hline Sample uptake: $60 \mathrm{sec}$. & Stabilize: 50 sec. \\
\hline \multicolumn{2}{|c|}{ Post run } \\
\hline Probe rinse (Sample): $40 \mathrm{sec}$. & Probe rinse (Standard): 40sec. \\
\hline
\end{tabular}

Based on the obtained results, the REEs were divided in two parts: major elements (scandium, yttrium, lanthanum, cerium, praseodymium, neodymium, samarium) and minor elements (europium, gadolinium, terbium, dysprosium, holmium, erbium, thulium, ytterbium, and lutetium). Two different analytical methods for extracted solutions were developed, one for major elements, situated in the range $20 \mu \mathrm{g} / \mathrm{L}$ to $100 \mu \mathrm{g} / \mathrm{L}$, and another one for minor elements, situated in the range $10 \mu \mathrm{g} / \mathrm{L}$ to 50 $\mu \mathrm{g} / \mathrm{L}$.

The following performance parameters were evaluated in the experimental test: detection limit (LOD), quantitation limit (LOQ), repeatability $\left(\mathrm{RSD}_{\mathrm{r}}\right)$, intermediate precision $\left(\mathrm{RSD}_{\mathrm{Ri}}\right)$, recovery and expanded uncertainty $\left(\mathrm{U}_{\mathrm{ex}}\right)$. The experimental studies applied in order to perform in-house validation for both methods are presented in Table 4.

Table 4. "in-house" validation experiments

\begin{tabular}{cc}
\hline Parameters & Experiments \\
\hline & Major elements \\
\hline LOQ and LOD & 5 independent fortified blank solutions $(5 \mu \mathrm{g} / \mathrm{L})$ \\
\hline Repeatability & 10 independent standard solution of $35 \mu \mathrm{g} / \mathrm{L}$ concentration \\
\hline Intermediate precision & 12 independent standard solution of $35 \mu \mathrm{g} / \mathrm{L}$ \\
\hline Recovery & 5 independent fortified sample solutions $(45 \mu \mathrm{L} / \mathrm{L})$ \\
\hline
\end{tabular}




\begin{tabular}{cc}
\hline & Minor elements \\
\hline LOQ and LOD & 5 independent fortified blank solutions $(3 \mu \mathrm{g} / \mathrm{L})$ \\
\hline Repeatability & 10 independent standard solution of $15 \mu \mathrm{g} / \mathrm{L}$ concentration \\
\hline Intermediate precision & 12 independent standard solution of $15 \mu \mathrm{g} / \mathrm{L}$ \\
\hline Recovery & 5 independent fortified sample solutions $(35 \mu \mathrm{g} / \mathrm{L})$ \\
\hline
\end{tabular}

The expanded uncertainty of the analytical results was estimated using the following formulas [26]:

$$
\begin{aligned}
& U_{\text {expanded }}=k * U_{\text {combined }} \\
& U_{\text {expanded }}=k * \sqrt{U_{c}^{2}+U_{v}^{2}+U_{\text {rep }}^{2}+\left(\frac{1}{U_{\text {mas }}}\right)^{2}+\left(\frac{1}{U_{\text {rec }}}\right)^{2}}
\end{aligned}
$$

where: $k$ is a coverage factor; value 2 for $95 \%$ confidence level;

$U_{\text {combined }}$ - combined standard uncertainty; $U_{c}$ - concentration uncertainty (instrument calibration, flasks, pipettes, reference standard material); $U_{v}-25-\mathrm{ml}$ volumetric flask (calibration, temperature); $U_{\text {rep }}$ - repeatability uncertainty (mass, volume, concentration, extraction recovery); $U_{\text {mass }}-$ weight uncertainty (balance calibration, linearity); $U_{r e c}-$ extraction recovery uncertainty.

The recovery $(\% \operatorname{Rec})$ is defined as:

$$
\% \operatorname{Rec}=\frac{C_{s p k}-C_{r e f}}{C_{a d d}} * 100
$$

where: $C_{s p k}$ is the analyte concentration in the spike sample; $C_{r e f}$ is the analyte concentration in the unfortified sample; $C_{a d d}$ is the analyte concentration in the added sample.

\section{Thermogravimetric analysis}

For structural characterization of the soil samples, a thermogravimetric analysis (TG) was performed, the TG operating parameters being presented in Table 5 .

Table 5. Thermogravimetric operating parameters

\begin{tabular}{cc}
\hline Parameters & Settings \\
\hline Temperature range & $35^{\circ} \mathrm{C}-1200^{\circ} \mathrm{C}$ \\
\hline Average flow rate (Argon- Carrier gas) & $60 \mathrm{~mL} / \mathrm{min}$ \\
\hline Average flow rate (Synthetic gas - Reaction gas) & $20 \mathrm{~mL} / \mathrm{min}$ \\
\hline Heating value & $10^{\circ} \mathrm{C} / \mathrm{min}$ \\
\hline
\end{tabular}

\section{Results and discussions}

\section{The physical-chemical characterization of soil samples}

Some specific analysis were performed for the physical-chemical characterization of the soil samples in order to highlight the influence of these characteristics on the tested mixtures extraction capacity for REEs.

Table 6. The physical-chemical characterization of soil samples

\begin{tabular}{ccccccccc}
\hline No. & Indicators & Units & P1 & P2 & P3 & P4 & P5 & P6 \\
\hline 1 & $\mathrm{pH}$ & $\mathrm{pH}$ unit. & 8.1 & 7.9 & 8.0 & 7.6 & 7.8 & 7.7 \\
\hline 2 & Conductivity & $\mu \mathrm{s} / \mathrm{cm}$ & 250 & 345 & 281 & 139 & 165 & 150 \\
\hline 3 & Total phosphorous & \% dry matter & 0.17 & 0.18 & 0.18 & 0.14 & 0.15 & 0.14 \\
\hline \multirow{2}{*}{$\begin{array}{c}\text { Total organic } \\
\text { carbon (TOC) }\end{array}$} & \% dry matter & 1.27 & 1.20 & 1.28 & 0.86 & 0.75 & 0.82 \\
\hline \multirow{2}{*}{5} & Total nitrogen $\left(\mathrm{N}_{\mathrm{t}}\right)$ & \% dry matter & 2.55 & 2.52 & 2.33 & 1.25 & 1.29 & 1.47 \\
\hline
\end{tabular}


As is reported in Table 6, a difference between the physical-chemical characterization of soils colected from Hunedoara County and Sibiu County. In this sense, was observed higher conductivity, TOC and total nitrogen were determined at P1, P2 and P3 (sampling sites from Hunedoara County), total nitrogen content being twice than in P4, P5 and P6 soil samples.

\section{Thermogravimetric analyses}

Thermogravimetric (TG) analysis was used to describe the decomposition of humic compounds from soil structure, weight transformations as a function of chemical composition being represented at different temperatures. TG analysis is important for obtaining information about the amount of organic matter released in the process and, also about the resulting residue which can be used in the study. Two different samples (P1, P4) were inserted into a crucible of alumina and analyzed. The P1 TG analysis is presented in Figure 3, the mass transformation during the analysis being presented in Table 7.

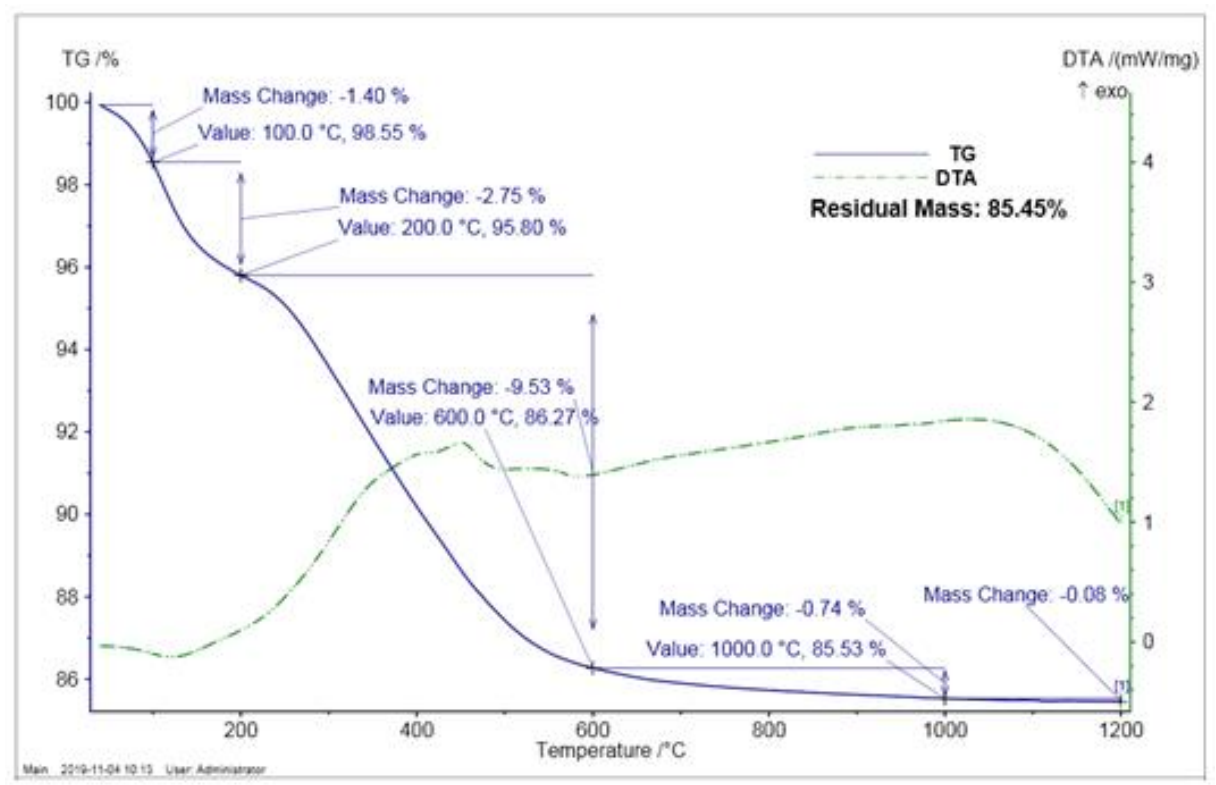

Figure 3. Thermogravimetric analysis of the P1 sample

At the end of the process, the residual mass for P1 sample was $85.45 \%$ (Figure 3), the highest content released in the process being correlated with humic mater (Table 7).

Table 7. Mass transformation during the tg analysis for $\mathrm{p} 1$ sample

\begin{tabular}{cccc}
\hline Step & Process & $\begin{array}{c}\text { Results } \\
{[\mathbf{\%}]}\end{array}$ & $\begin{array}{c}\text { Temperature } \\
{\left[{ }^{\circ} \mathbf{C}\right]}\end{array}$ \\
\hline $\mathbf{1}$ & Evaporation of hydroscopic moisture & 1.40 & $100^{\circ} \mathrm{C}$ \\
\hline & $\begin{array}{c}\text { Decomposition of less condensed peat compounds: cellulose and other } \\
\text { polysaccharides, external }\end{array}$ & 2.75 & $200^{\circ} \mathrm{C}$ \\
$\mathbf{2}$ & functional groups of humic matter, aliphatic compounds & & \\
\hline $\mathbf{3}$ & Decomposition of more condensed compounds: aromatic structure of \\
lignin and humic mater & 9.53 & \\
\hline $\mathbf{4}$ & Decomposition of volatile substances and the carbonates & 0.74 & $1000^{\circ} \mathrm{C}$ \\
\hline
\end{tabular}

The TG analysis of P4 sample is presented in Figure 4, the mass transformation during the analysis being presented in Table 8 . 


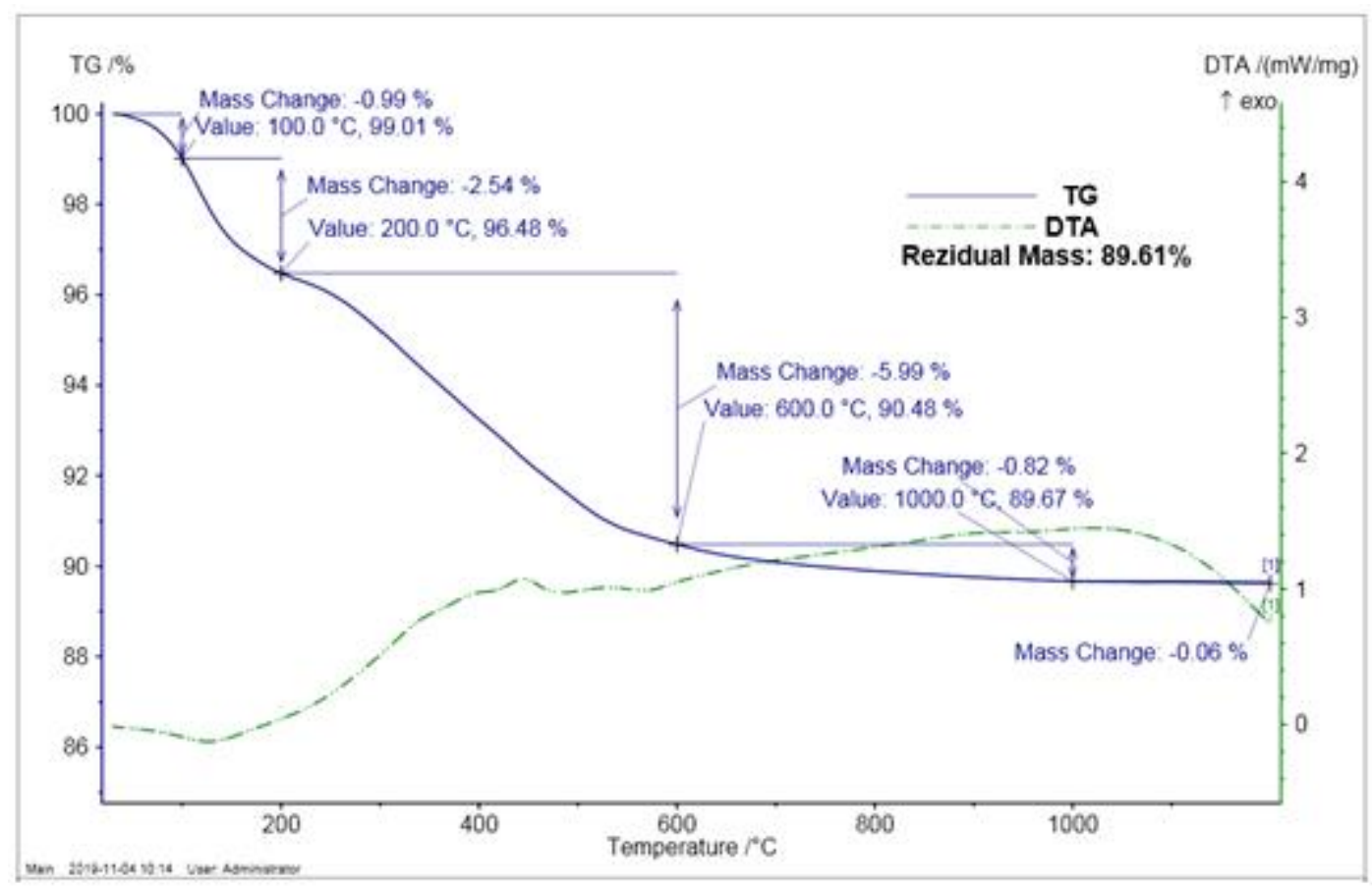

Figure 4. Thermogravimetric analysis of the P4 sample

Table 8. Mass transformation during the tg analysis for $\mathrm{p} 2$ sample

\begin{tabular}{cccc}
\hline Step & Process & $\begin{array}{c}\text { Results } \\
{[\mathbf{\%}]}\end{array}$ & $\begin{array}{c}\text { Temperature } \\
{\left[{ }^{\mathbf{0}} \mathbf{C}\right]}\end{array}$ \\
\hline $\mathbf{1}$ & Evaporation of hydroscopic moisture & 0.99 & $100^{\circ} \mathrm{C}$ \\
\hline & $\begin{array}{c}\text { Decomposition of less condensed peat compounds: cellulose and other } \\
\text { polysaccharides, external }\end{array}$ & 2.54 & $200^{\circ} \mathrm{C}$ \\
$\mathbf{2}$ & functional groups of humic matter, aliphatic compounds & & $600^{\circ} \mathrm{C}$ \\
\hline $\mathbf{3}$ & Decomposition of more condensed compounds: aromatic structure of \\
lignin and humic mater & 5.99 & $1000^{\circ} \mathrm{C}$ \\
\hline
\end{tabular}

For P4 sample, the residual mass remained at the end of the process was $89.61 \%$ (figure 4 ), the highest content released being also humic mater compounds, reported as possible interferences in REEs analyzes [27]. The percentage of mass released in both investigated samples was lower than $15 \%$.

\section{Performance parameters for REEs in-house validation methods}

In order to validate the proposed methods, experimental tests were performed on $\mathrm{P} 4$ sample, due to high content of REEs (tables 9, 10).

Table 9. Performance parameters for rees major elements

\begin{tabular}{llllllll}
\hline Element & $\begin{array}{l}\text { LOD } \\
(\mathbf{m g} / \mathbf{k g})\end{array}$ & $\begin{array}{l}\mathbf{L O Q} \\
(\mathbf{m g} / \mathbf{k g})\end{array}$ & $\begin{array}{l}\text { Accuracy } \\
(\mathbf{m g} / \mathbf{k g})\end{array}$ & $\begin{array}{l}\mathbf{R S D}_{\mathbf{r}}{ }^{*} \\
(\mathbf{\%})\end{array}$ & $\begin{array}{l}\mathbf{R S D}_{\mathbf{R}}{ }^{* *} \\
(\boldsymbol{\%})\end{array}$ & $\begin{array}{l}\text { Recovery } \\
(\mathbf{\%})\end{array}$ & $\begin{array}{l}\mathbf{U}_{\mathbf{e x}} * * * \\
(\mathbf{\%})\end{array}$ \\
\hline Scandium & 0.028 & 0.09 & 0.051 & 1.04 & 1.58 & 92.04 & 17.04 \\
\hline Ytrium & 0.016 & 0.05 & 0.043 & 1.13 & 1.74 & 96.04 & 17.77 \\
\hline Lantanum & 0.011 & 0.04 & 0.026 & 0.74 & 1.11 & 99.18 & 15.09 \\
\hline Cerium & 0.010 & 0.03 & 0.024 & 0.93 & 1.22 & 99.90 & 15.14 \\
\hline Praseodim & 0.016 & 0.05 & 0.031 & 1.66 & 2.39 & 86.13 & 17.61 \\
\hline Neodinum & 0.018 & 0.06 & 0.043 & 1.04 & 2.46 & 89.89 & 18.87 \\
\hline Samarium & 0.021 & 0.07 & 0.041 & 1.10 & 1.84 & 91.55 & 16.17 \\
\hline
\end{tabular}

*Repeatability; **Intermediate precision; ***Expanded uncertainty 
The quality control of the results, due to the lack of a commercial certified reference material (CRM) for soil matrix with rare metals, was achieved with a fortified soil sample.

Minor elements were added in the concentrations of $35 \mu \mathrm{g} / \mathrm{L}$ while the added solution for major elements was $45 \mu \mathrm{g} / \mathrm{L}$. The recovery percentages were calculated, highest values for major elements were recorded for cerium (99.90\%), lanthanum (99.18\%), yttrium (96.04\%), scandium (92.04\%) and samarium $(91.55 \%)$, Table 9.

Table 10. Performance parameters for rees minor elements

\begin{tabular}{|c|c|c|c|c|c|c|c|}
\hline Element & $\begin{array}{l}\mathrm{LOD} \\
(\mathrm{mg} / \mathrm{kg})\end{array}$ & $\begin{array}{l}\text { LOQ } \\
(\mathrm{mg} / \mathrm{kg})\end{array}$ & $\begin{array}{l}\text { Accuracy } \\
\text { (mg/kg) }\end{array}$ & $\begin{array}{l}\text { RSD }^{*} * \\
(\%)\end{array}$ & $\begin{array}{l}\text { RSD }{ }^{* * *} \\
(\%)\end{array}$ & $\begin{array}{l}\text { Recovery } \\
(\%)\end{array}$ & $\begin{array}{l}\mathrm{Uex}_{\mathrm{ex} * *} \\
(\%)\end{array}$ \\
\hline Europium & 0.021 & 0.07 & 0.035 & 1.27 & 1.68 & 85.90 & 19.07 \\
\hline Gadolinium & 0.025 & 0.08 & 0.025 & 1.65 & 2.06 & 86.56 & 18.89 \\
\hline Terbium & 0.020 & 0.07 & 0.064 & 1.09 & 1.85 & 85.23 & 19.02 \\
\hline Dysprosium & 0.024 & 0.08 & 0.063 & 1.51 & 2.07 & 85.97 & 18.89 \\
\hline Holmium & 0.022 & 0.07 & 0.061 & 1.29 & 1.83 & 86.21 & 18.99 \\
\hline Erbium & 0.026 & 0.09 & 0.064 & 1.21 & 1.68 & 85.22 & 18.86 \\
\hline Tulium & 0.024 & 0.08 & 0.075 & 1.54 & 2.20 & 85.51 & 18.92 \\
\hline Yterbium & 0.027 & 0.09 & 0.080 & 1.35 & 2.19 & 85.85 & 18.88 \\
\hline Lutetium & 0.025 & 0.08 & 0.72 & 1.78 & 2.13 & 86.65 & 18.96 \\
\hline
\end{tabular}

The mean recovery percentage for minor elements was situated around $86 \%$ value $(85.80 \% \pm$ $0.41 \%)$, being lower than the one for major elements $(93.53 \% \pm 4.12 \%)$. Determined values for performance parameters were situated in the accepted range according to literature indication [25].

\section{Metallic elements analyzes}

In table 11 are presented the obtained values for $\mathrm{As}, \mathrm{Cd}, \mathrm{Cr}, \mathrm{Mn}, \mathrm{Ni}, \mathrm{Pb}$ and $\mathrm{Zn}$ from soil samples (initial sample, figure 2), the results being within the reference values for soil quality according to Romanian Order 756/1997 [28].

Table 11. Metal concentrations in initial samples

\begin{tabular}{|c|c|c|c|c|c|c|c|}
\hline \multirow[t]{2}{*}{ Element } & \multicolumn{7}{|c|}{ Content (mg/kg dry matter) } \\
\hline & As & Cd & $\mathrm{Cr}$ & Mn & $\mathbf{N i}$ & $\mathbf{P b}$ & Zn \\
\hline P1 & $1.95 \pm 0.35$ & $0.35 \pm 0.07$ & $20.1 \pm 1.96$ & $660 \pm 27$ & $19.9 \pm 1.98$ & $15.2 \pm 1.54$ & $70.5 \pm 5.08$ \\
\hline P2 & $1.89 \pm 0.33$ & $0.30 \pm 0.06$ & $20.6 \pm 2.01$ & $671 \pm 27$ & $18.5 \pm 1.84$ & $14.2 \pm 1.44$ & $75.6 \pm 5.45$ \\
\hline $\mathbf{P 3}$ & $1.96 \pm 0.34$ & $0.34 \pm 0.07$ & $20.5 \pm 2.00$ & $667 \pm 27$ & $19.0 \pm 1.89$ & $14.2 \pm 1.44$ & $72.7 \pm 5.24$ \\
\hline P4 & $1.39 \pm 0.24$ & $0.17 \pm 0.04$ & $14.8 \pm 1.44$ & $521 \pm 21$ & $11.8 \pm 1.18$ & $8.24 \pm 0.84$ & $55.3 \pm 3.98$ \\
\hline P5 & $1.43 \pm 0.25$ & $0.20 \pm 0.04$ & $16.7 \pm 1.63$ & $540 \pm 22$ & $13.2 \pm 1.32$ & $9.69 \pm 0.98$ & $58.2 \pm 4.19$ \\
\hline P6 & $1.24 \pm 0.21$ & $0.18 \pm 0.04$ & $15.1 \pm 1.47$ & $546 \pm 22$ & $12.9 \pm 1.29$ & $9.35 \pm 0.95$ & $57.6 \pm 4.15$ \\
\hline
\end{tabular}

To assess the quality control of the analytical results of metallic elements $\mathrm{As}, \mathrm{Cd}, \mathrm{Cr}, \mathrm{Mn}, \mathrm{Ni}, \mathrm{Pb}$, $\mathrm{Zn}$, a certificate reference material CRM ERM-CC 141 Loam Soil was analysed. The recovery percentage were situated in the range 90.2 to 99.5 , as is indicated in Table 12 .

Table 12. Results and revovery for loam soil crm erm-cc 141

\begin{tabular}{cccc}
\hline Element & CRM certificate $(\mathbf{m g} / \mathbf{k g})$ & CRM obtained $\mathbf{~ m g} / \mathbf{k g})$ & Recovery \% \\
\hline Arsenic & $9.9 \pm 1.5$ & $9.81 \pm 1.7$ & 90.2 \\
\hline Cadmium & $0.35 \pm 0.05$ & $0.34 \pm 0.07$ & 90.5 \\
\hline Chromium & $86 \pm 8$ & $85.2 \pm 8.3$ & 92.1 \\
\hline Manganese & $464 \pm 18$ & $466.3 \pm 19$ & 99.3 \\
\hline Nickel & $26.4 \pm 2.4$ & $27.1 \pm 2.7$ & 99.5 \\
\hline Lead & $41 \pm 4$ & $42.4 \pm 4.3$ & 97.6 \\
\hline Zinc & $57 \pm 4$ & $58.3 \pm 4.2$ & 99.1 \\
\hline
\end{tabular}




\section{REEs analyses}

All the soil samples were analysed in duplicate, the results presented in tables 13-15 represent the mean values.

Table 13. Major REEs concentration obtained with digestion method $\mathrm{i}$

\begin{tabular}{|c|c|c|c|c|c|c|c|c|}
\hline \multirow{2}{*}{$\begin{array}{c}\text { Sample } \\
\text { code }\end{array}$} & \multirow[t]{2}{*}{ Samples } & \multicolumn{7}{|c|}{ Analyzed element [mg/kg] } \\
\hline & & Sc & $\mathbf{Y}$ & La & $\mathrm{Ce}$ & Pr & Nd & Sm \\
\hline \multirow{4}{*}{ P1 } & Initial & $2.11 \pm 0.36$ & $0.13 \pm 0.02$ & $2.15 \pm 0.32$ & $1.02 \pm 0.15$ & $<0.05$ & $<0.06$ & $<0.07$ \\
\hline & $550^{\circ} \mathrm{C}$ & $5.33 \pm 0.90$ & $0.30 \pm 0.05$ & $4.66 \pm 0.70$ & $5.64 \pm 0.85$ & $0.10 \pm 0.02$ & $0.16 \pm 0.03$ & $0.21 \pm 0.03$ \\
\hline & $700^{\circ} \mathrm{C}$ & $9.78 \pm 1.67$ & $0.42 \pm 0.08$ & $9.13 \pm 1.38$ & $18.2 \pm 2.76$ & $0.11 \pm 0.02$ & $0.22 \pm 0.04$ & $0.55 \pm 0.09$ \\
\hline & $850^{\circ} \mathrm{C}$ & $4.20 \pm 0.72$ & $0.27 \pm 0.05$ & $7.11 \pm 1.07$ & $8.00 \pm 1.21$ & $0.09 \pm 0.02$ & $0.17 \pm 0.03$ & $0.19 \pm 0.03$ \\
\hline \multirow{4}{*}{ P2 } & Initial & $1.96 \pm 0.33$ & $0.12 \pm 0.02$ & $2.10 \pm 0.32$ & $1.00 \pm 0.15$ & $<0.05$ & $<0.06$ & $<0.07$ \\
\hline & $550^{\circ} \mathrm{C}$ & $5.10 \pm 0.87$ & $0.25 \pm 0.05$ & $3.70 \pm 0.56$ & $4.86 \pm 0.74$ & $0.10 \pm 0.02$ & $0.11 \pm 0.02$ & $0.18 \pm 0.03$ \\
\hline & $700^{\circ} \mathrm{C}$ & $8.46 \pm 1.44$ & $0.33 \pm 0.06$ & $8.46 \pm 1.28$ & $17.2 \pm 2.60$ & $0.08 \pm 0.01$ & $0.20 \pm 0.04$ & $0.44 \pm 0.07$ \\
\hline & $850^{\circ} \mathrm{C}$ & $3.13 \pm 0.53$ & $0.21 \pm 0.04$ & $6.74 \pm 1.02$ & $6.85 \pm 1.04$ & $0.09 \pm 0.01$ & $0.11 \pm 0.02$ & $0.12 \pm 0.02$ \\
\hline \multirow{4}{*}{ P3 } & Initial & $1.26 \pm 0.21$ & $0.11 \pm 0.02$ & $2.12 \pm 0.32$ & $1.00 \pm 0.15$ & $<0.05$ & $<0.06$ & $<0.07$ \\
\hline & $550^{\circ} \mathrm{C}$ & $4.86 \pm 0.83$ & $0.29 \pm 0.05$ & $3.87 \pm 0.58$ & $5.15 \pm 0.78$ & $0.08 \pm 0.01$ & $0.14 \pm 0.03$ & $0.19 \pm 0.03$ \\
\hline & $700^{\circ} \mathrm{C}$ & $9.11 \pm 1.55$ & $0.41 \pm 0.07$ & $8.99 \pm 1.36$ & $17.9 \pm 2.71$ & $0.10 \pm 0.02$ & $0.20 \pm 0.04$ & $0.51 \pm 0.08$ \\
\hline & $850^{\circ} \mathrm{C}$ & $3.76 \pm 0.64$ & $0.21 \pm 0.04$ & $6.12 \pm 0.92$ & $6.68 \pm 1.01$ & $0.10 \pm 0.02$ & $0.16 \pm 0.03$ & $0.18 \pm 0.03$ \\
\hline \multirow{4}{*}{ P4 } & Initial & $0.60 \pm 0.10$ & $0.49 \pm 0.09$ & $5.99 \pm 0.90$ & $7.37 \pm 1.12$ & $0.15 \pm 0.03$ & $0.23 \pm 0.04$ & $0.22 \pm 0.04$ \\
\hline & $550^{\circ} \mathrm{C}$ & $11.2 \pm 1.90$ & $1.05 \pm 0.19$ & $9.83 \pm 1.48$ & $14.7 \pm 2.22$ & $0.37 \pm 0.07$ & $0.33 \pm 0.06$ & $0.69 \pm 0.11$ \\
\hline & $7^{\circ 00^{\circ} \mathrm{C}}$ & $15.1 \pm 2.57$ & $1.09 \pm 0.19$ & $19.7 \pm 2.98$ & $31.1 \pm 4.71$ & $0.58 \pm 0.10$ & $0.52 \pm 0.10$ & $0.84 \pm 0.14$ \\
\hline & $850^{\circ} \mathrm{C}$ & $7.86 \pm 1.34$ & $1.02 \pm 0.18$ & $9.51 \pm 1.43$ & $12.1 \pm 1.83$ & $0.16 \pm 0.03$ & $0.13 \pm 0.02$ & $0.26 \pm 0.04$ \\
\hline \multirow{4}{*}{ P5 } & Initial & $0.43 \pm 0.07$ & $0.41 \pm 0.07$ & $5.13 \pm 0.77$ & $7.23 \pm 1.09$ & $0.14 \pm 0.03$ & $0.22 \pm 0.04$ & $0.22 \pm 0.04$ \\
\hline & $550^{\circ} \mathrm{C}$ & $10.3 \pm 1.75$ & $1.03 \pm 0.18$ & $9.82 \pm 1.5$ & $14.7 \pm 2.22$ & $0.36 \pm 0.06$ & $0.31 \pm 0.06$ & $0.65 \pm 0.11$ \\
\hline & $700^{\circ} \mathrm{C}$ & $14.9 \pm 2.5$ & $1.06 \pm 0.19$ & $19.7 \pm 2.97$ & $28.7 \pm 4.34$ & $0.53 \pm 0.09$ & $0.49 \pm 0.09$ & $0.81 \pm 0.13$ \\
\hline & $850^{\circ} \mathrm{C}$ & $7.8 \pm 1.3$ & $1.00 \pm 0.18$ & $9.48 \pm 1.48$ & $11.4 \pm 1.72$ & $0.15 \pm 0.03$ & $0.12 \pm 0.02$ & $0.22 \pm 0.04$ \\
\hline \multirow{4}{*}{ P6 } & Initial & $0.56 \pm 0.09$ & $0.49 \pm 0.09$ & $5.91 \pm 0.89$ & $7.24 \pm 1.10$ & $0.14 \pm 0.03$ & $0.22 \pm 0.04$ & $0.22 \pm 0.03$ \\
\hline & $550^{\circ} \mathrm{C}$ & $10.6 \pm 1.8$ & $1.01 \pm 0.18$ & $9.75 \pm 1.47$ & $14.0 \pm 2.12$ & $0.35 \pm 0.06$ & $0.31 \pm 0.06$ & $0.62 \pm 0.10$ \\
\hline & $700^{\circ} \mathrm{C}$ & $15.0 \pm 2.6$ & $1.02 \pm 0.18$ & $18.6 \pm 2.81$ & $30.8 \pm 4.67$ & $0.56 \pm 0.10$ & $0.50 \pm 0.09$ & $0.82 \pm 0.13$ \\
\hline & $850^{\circ} \mathrm{C}$ & $7.4 \pm 1.3$ & $1.00 \pm 0.18$ & $8.59 \pm 1.30$ & $11.4 \pm 1.72$ & $0.13 \pm 0.02$ & $0.10 \pm 0.02$ & $0.21 \pm 0.03$ \\
\hline
\end{tabular}

The results for REEs major elements (extraction method I) were reported in all analysed soil samples (initial samples, residues at $550^{\circ} \mathrm{C}, 700^{\circ} \mathrm{C}$, respectively $800^{\circ} \mathrm{C}$ ), as is presented in table 13 . The results indicate that the content of humic compounds from soil samples influence the extraction of REEs, the samples with highest content of organic compounds having the lowest REEs values (initial sample), and so only approximately $30 \%$ of REEs content was extracted. The highest values of REEs were obtained for residues calcinated at $700^{\circ} \mathrm{C}$. The explanation of this behaviour probably is represented by the uncomplete decomposition of organic compounds at $550^{\circ} \mathrm{C}(40 \%$ to $85 \%$ recovery for different elements) and REEs loses at $850^{\circ} \mathrm{C}$ high temperature (32\% to $71 \%$ lower recovery).

Highest REEs values were obtained for cerium, lanthanum and scandium in P4, P5 and P6 samples, concentration increase being the following: $\mathrm{Ce}>\mathrm{La}>\mathrm{Sc}>\mathrm{Y}>\mathrm{Sm}>\mathrm{Pr}>\mathrm{Nd}$ (Table 13).

Table 14. Minor REEs concentration obtained with digestion method i at $700^{\circ} \mathrm{C}$

\begin{tabular}{|c|c|c|c|c|c|c|c|c|c|}
\hline \multirow[t]{2}{*}{ Sample code } & \multicolumn{9}{|c|}{ Analyzed element [mg/kg] } \\
\hline & Eu & Gd & $\mathbf{T b}$ & Dy & Ho & Er & Tm & $\mathbf{Y b}$ & Lu \\
\hline P1 & $\begin{array}{l}\mathbf{0 . 1 3} \\
\pm 0.02\end{array}$ & $\begin{array}{l}\mathbf{0 . 1 9} \\
\pm 0.04\end{array}$ & $\begin{array}{l}\mathbf{0 . 1 2} \\
\pm 0.02\end{array}$ & $<0.08$ & $<0.07$ & $<0.09$ & $<0.08$ & $<0.09$ & $<0.08$ \\
\hline $\mathbf{P 2}$ & $\begin{array}{l}\mathbf{0 . 1 1} \\
\pm 0.02\end{array}$ & $\begin{array}{l}\mathbf{0 . 1 6} \\
\pm 0.03\end{array}$ & $\begin{array}{l}\mathbf{0 . 1 0} \\
\pm 0.02\end{array}$ & $<0.08$ & $<0.07$ & $<0.09$ & $<0.08$ & $<0.09$ & $<0.08$ \\
\hline P3 & $\begin{array}{l}\mathbf{0 . 0 9} \\
\pm 0.02 \\
\end{array}$ & $\begin{array}{l}\mathbf{0 . 1 8} \\
\pm 0.03 \\
\end{array}$ & $\begin{array}{l}\mathbf{0 . 1 1} \\
\pm 0.02 \\
\end{array}$ & $<0.08$ & $<0.07$ & $<0.09$ & $<0.08$ & $<0.09$ & $<0.08$ \\
\hline $\mathbf{P 4}$ & $\begin{array}{l}\mathbf{0 . 1 8} \\
\pm 0.03\end{array}$ & $\begin{array}{l}\mathbf{0 . 2 7} \\
\pm 0.05\end{array}$ & $\begin{array}{l}\mathbf{0 . 1 3} \\
\pm 0.02\end{array}$ & $\begin{array}{l}\mathbf{0 . 1 5} \\
\pm 0.03\end{array}$ & $\begin{array}{l}\mathbf{0 . 1 3} \\
\pm 0.02\end{array}$ & $\begin{array}{l}\mathbf{0 . 1 4} \\
\pm 0.03\end{array}$ & $\begin{array}{l}\text { 0.18 } \\
\pm 0.03\end{array}$ & $\begin{array}{l}\mathbf{0 . 1 1} \\
\pm 0.02\end{array}$ & $\begin{array}{l}\mathbf{0 . 1 2} \\
\pm 0.02 \\
\end{array}$ \\
\hline P5 & $\begin{array}{l}\mathbf{0 . 1 6} \\
\pm 0.03\end{array}$ & $\begin{array}{l}\mathbf{0 . 2 2} \\
\pm 0.04\end{array}$ & $\begin{array}{l}\mathbf{0 . 0 9} \\
\pm 0.02\end{array}$ & $\begin{array}{l}\mathbf{0 . 1 2} \\
\pm 0.02\end{array}$ & $\begin{array}{l}\mathbf{0 . 1 1} \\
\pm 0.02\end{array}$ & $\begin{array}{l}\mathbf{0 . 1 2} \\
\pm 0.02\end{array}$ & $\begin{array}{l}\mathbf{0 . 1 0} \\
\pm 0.02\end{array}$ & $\begin{array}{l}\mathbf{0 . 1 0} \\
\pm 0.02\end{array}$ & $\begin{array}{l}\mathbf{0 . 1 0} \\
\pm 0.02\end{array}$ \\
\hline P6 & $\begin{array}{l}\mathbf{0 . 1 5} \\
\pm 0.03\end{array}$ & $\begin{array}{l}\mathbf{0 . 2 1} \\
\pm 0.04\end{array}$ & $\begin{array}{l}\mathbf{0 . 1 1} \\
\pm 0.02\end{array}$ & $\begin{array}{l}\mathbf{0 . 1 3} \\
\pm 0.02\end{array}$ & $\begin{array}{l}\mathbf{0 . 0 9} \\
\pm 0.02\end{array}$ & $\begin{array}{l}\text { 0.11 } \\
\pm 0.02\end{array}$ & $\begin{array}{l}\mathbf{0 . 0 9} \\
\pm 0.02\end{array}$ & $\begin{array}{l}\mathbf{0 . 0 9} \\
\pm 0.02\end{array}$ & $\begin{array}{l}\mathbf{0 . 0 9} \\
\pm 0.02\end{array}$ \\
\hline
\end{tabular}


Regarding REEs minor elements, the results obtained with digestion method I from initial samples, residues at $550^{\circ} \mathrm{C}$ and $800^{\circ} \mathrm{C}$ were situated below quantification limits for all investigated elements (Eu, Gd, Tb, Dy, Ho, Er, Tm, Yb, Lu). Same observation as in major elements case could be stated, that organic compounds interfere in REEs determination.

In Table 14 are reported only the values of REEs obtained from $700^{\circ} \mathrm{C}$ residue, the concentrations being either under quantification limits or at very small values, in the range $0.09 \mathrm{mg} / \mathrm{kg}$ to $0.27 \mathrm{mg} / \mathrm{kg}$. In samples collected from Hunedoara County (P1-P3), Dy, Ho, Er, Tm, Yb and Lu concentrations were under the LOQ, in contrast, with the soil samples from Sibiu County (P4-P6) were the values were small, but above the LOQ.

Table 15 presents the major elements results obtained with digestion method II, extraction of REEs from $700^{\circ} \mathrm{C}$ residues with aqua regia mixture (usually used method for metal extraction).

Table 15. Major REEs concentration obtained with digestion method ii at $700^{\circ} \mathrm{C}$

\begin{tabular}{cccccccc}
\hline $\begin{array}{c}\text { Sample } \\
\text { code }\end{array}$ & \multicolumn{7}{c}{ Analyzed element $[\mathbf{m g} / \mathbf{k g}]$} \\
\cline { 2 - 8 } Sc & Y & La & Ce & Pr & Nd & Sm \\
\hline P1 & $2.12 \pm 0.36$ & $0.12 \pm 0.02$ & $1.15 \pm 0.17$ & $3.8 \pm 0.57$ & $0.09 \pm 0.02$ & $0.10 \pm 0.02$ & $0.12 \pm 0.02$ \\
\hline P2 & $2.00 \pm 0.34$ & $0.09 \pm 0.02$ & $0.10 \pm 0.01$ & $3.1 \pm 0.47$ & $0.07 \pm 0.01$ & $0.08 \pm 0.02$ & $0.10 \pm 0.02$ \\
\hline P3 & $1.75 \pm 0.30$ & $0.08 \pm 0.01$ & $0.09 \pm 0.01$ & $2.8 \pm 0.42$ & $0.07 \pm 0.01$ & $0.08 \pm 0.01$ & $0.09 \pm 0.02$ \\
\hline P4 & $6.55 \pm 1.12$ & $0.15 \pm 0.03$ & $7.99 \pm 1.21$ & $18.1 \pm 2.74$ & $0.13 \pm 0.02$ & $0.13 \pm 0.02$ & $0.14 \pm 0.02$ \\
\hline P5 & $6.11 \pm 1.04$ & $0.13 \pm 0.02$ & $7.11 \pm 1.07$ & $18.0 \pm 2.72$ & $0.11 \pm 0.02$ & $0.12 \pm 0.02$ & $0.12 \pm 0.02$ \\
\hline P6 & $5.99 \pm 1.02$ & $0.14 \pm 0.03$ & $6.79 \pm 1.02$ & $17.6 \pm 2.65$ & $0.10 \pm 0.02$ & $0.09 \pm 0.02$ & $0.11 \pm 0.02$ \\
\hline
\end{tabular}

Reported results are lower than the one obtained with digestion method I. One reason for this behavior could be the interference of chlorine ions with the argon in the ICP-MS technique.

To highlight the differences between the extraction methods results (table 13 and 15), a report of REEs major elements concentration obtained with method II (aqua regia) was divided to REEs major elements concentration resulted from method I (nitric acid and hydrogen peroxide) and plotted in Figure 5.

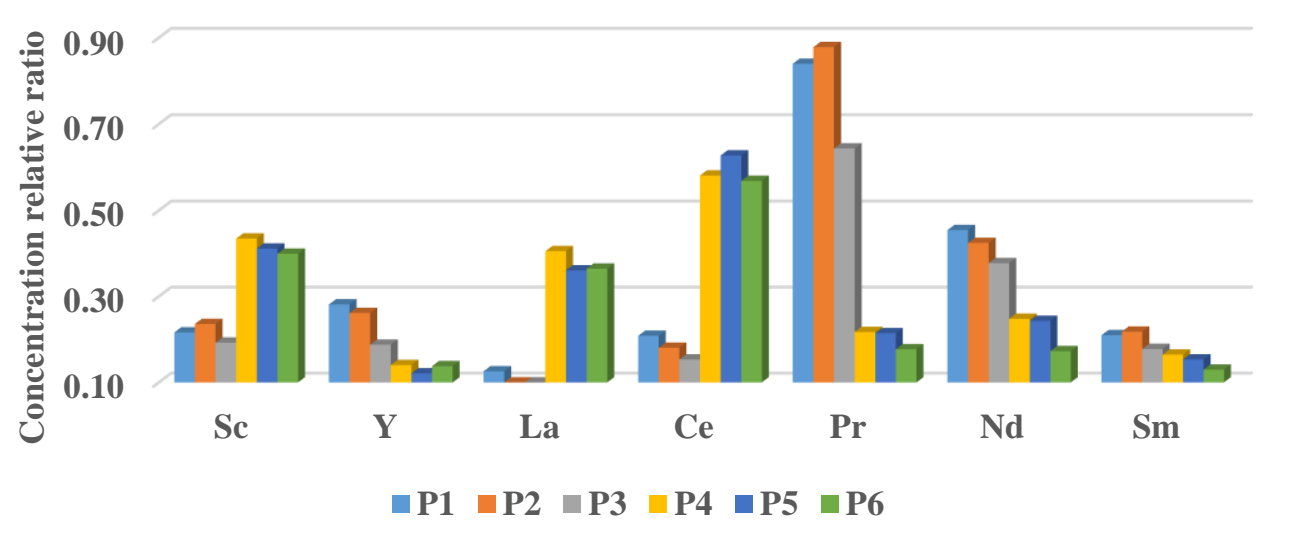

Figure 5. The concentration relative ratio of REEs major elements for two different extraction methods applied to $700^{\circ} \mathrm{C}$ residue, [REEs mg/kg, method II]/[ REEs mg/kg, method I]

The behavior of soils in aqua regia extraction was different, so it was observed that the selected soils from Hunedoara County have a structure that allows extraction of a larger quantity of yttrium, praseodymium and neodymium. Instead, more quantity of scandium, lanthanum and cerium were extracted with aqua regia from Sibiu County soils. There are no significant differences regarding the samarium concentration.

The results for all minor elements analyzed ( $\mathrm{Eu}, \mathrm{Gd}, \mathrm{Tb}, \mathrm{Dy}, \mathrm{Ho}, \mathrm{Er}, \mathrm{Tm}, \mathrm{Yb}, \mathrm{Lu})$, extracted with aqua regia from $700^{\circ} \mathrm{C}$ residues, were situated below the quantification limits. 


\section{Conclusions}

Lanthanides (lanthanum, cerium, praseodymium, neodymium, samarium, europium, gadolinium, terbium, dysprosium, holmium, erbium, thulium, ytterbium, lutetium), scandium and yttrium from soil samples were quantified using ICP-MS technique after a thermal treatment of soil at $700^{\circ} \mathrm{C}$ and extraction of REEs with a mixture of nitric acid and hydrogen peroxide.

Simultaneous analytical methods for measurement of REEs major elements ( $\mathrm{Sc}, \mathrm{Y}, \mathrm{La}, \mathrm{Ce}, \mathrm{Pr}, \mathrm{Nd}$, $\mathrm{Sm}$ ), respectively REEs minor elements (Eu, Gd, Tb, Dy, Ho, Er, Tm, Yb, Lu) were in-house validated, performance parameters such as: detection limit, quantitation limit, repeatability, intermediate precision, recovery and expanded uncertainty were evaluated. The methods proved to be reliable, sensitive, selective, allowing rapid determination of rare earth elements from soils.

Developed methods were applied on six soil samples, collected from Hunedoara and Sibiu Counties, Romania. The results indicated that for $\mathrm{Sc}, \mathrm{La}$ and $\mathrm{Ce}$ were recorded highest values, scandium range concentration being situated between $8.5 \mathrm{mg} / \mathrm{kg}$ to $15 \mathrm{mg} / \mathrm{kg}$ and lanthanum concentration domain being $8.5 \mathrm{mg} / \mathrm{kg}$ to $19.7 \mathrm{mg} / \mathrm{kg}$. Cerium values were within the range 17.2 $\mathrm{mg} / \mathrm{kg}$ to $31.1 \mathrm{mg} / \mathrm{kg}$. The recorded values are situated within the normal limits for these elements in the Earth's crust.

Acknowledgements:This work was carried out through Nucleu Program financed by the Ministry of Research and Innovation, contract no. 20N/2019, project code PN 19040401.

\section{References}

1. VUKOJEVIC V., DURDIC S., STEFANOVIC V., TRIFKOVIC J., CAKMAK D., PEROVIC V., MUTIĆ J., Environ. Sci. Pollut. Res. Int., 26, 2019, p.5422.

2. KARCZEWSKA, A., LEWIŃSKA, K., SIEPAK, M., GAŁKA, B., Pol. J. Environ. Stud. 28, no. 3, 2019, p. 1517.

3. BAU, M., SCHMIDT, K., PACK, A., BENDEL, V., KRAEMER, D., Appl. Geochem., 90, 2018, p.142.

4. FALANDYSZ, J., SAPKOTA, A., MĘDYK, M., FENG, X., Food Chem., 221, 2017b, p.24

5. LADONIN, D.V., Eurasian. Soil Science. 50, no. 6, 2017, p. 672.

6. MIGASZEWSKI, Z.M., GAŁUSZKA, A., Crit. Rev. Environ. Sci. Tech., 45, no.5, 2015, p.429.

7. ROMERO-FREIRE, A., MINGUEZ, L., PELLETIER, M., Sci. Total Environ., 612, 2018, p.831.

8. DOŁĘGOWSKA, S., MIGASZEWSKI, Z.M., Environ. Pollut., 178, 2013, p.33.

9. IORDACHE, P.Z., SOMOGHI V, SAVU, I, PETREA, N., MITRU, G., PETRE, R., DIONEZIE, B., ORDEANU, V., KIM, L., MUTIHAC, L., J. Optoelectron. Adv. M., 2, no. 8, 2008, p. 491.

10. HIRTOPANU, P., ANDERSEN, J.C., FAIRHURST, R.J., JAKAB, G., Proc. Rom. Acad. 15, 2013a, p.59

11. CHEN J., YANG R., J. Rare Earths, 28, 2010, p.517.

12. LI, X., CHEN, Z., CHEN, Z., ZHANG, Y., Chemosphere, 93, no. 6, 2013, p.1240.

13. SILVA, M.M.V.G., LOPES, S.P., GOMES, E.C., Chem. Erde-Geochem., 74, 2014, p.545.

14. REN, Y, REN, X, MA, J, YAN, L, J., Rare Earths, 34, 2016, p.638.

15. ZHENG, S., ZHANG, C., SHI, K., WANG, J., SUN, G., HU, Q., J. Rare Earth, 36, 2018, p.331.

16. U.S. GEOLOGICAL SURVEY. MINERALS YEAR BOOK-RARE EARTHS. 2017. <https://minerals.usgs.gov/minerals/pubs/commodity/rare earths/mcs-2017-raree.pdf $>$.

17. HOWER, C.J., QIAN, D., BRIOT, N. J., SANTILLAN-JIMENEZ, E., HOOD, M.M., TAGGART, R. K., AND HSU-KIM, H., Minerals., 206, no.9, 2019, p.1

18. FOLGUERAS, M. B., ALONSO, M., FOLGUERAS, J.R., AND LAGE, S., Proceedings. MDPI, 1462, no. 2, 2018, p. 1

19. RIVERA, R.M., XAKALASHE, B., OUNOUGHENE, G., BINNEMANS, K., FRIEDRICH, B., VAN GERVEN, T., Hydrometallurgy, 184, 2019, p.162 
20. KIM, L., VASILE, G.G., STANESCU, B., CALINESCU, S., BATRINESCU, G., J. Environ. Prot. Ecol., 16, no. 4, 2015, p.1227

21. KIM, L., CATRINA (TRAISTARU), G.A., STANESCU, B., PASCU, L.F., GHEORGHITA, T., MANOLACHE, D., Rev. Chim., 70, (1), 2019, 269

22. VASIlE, G.G., DINU, C., KIM, L., TENEA, A., SIMION, M., ENE, C., SPINU, C., UNGUREANU, E-M., MANOLACHE, D., Rev. Chim., 70, (1), 2019, 286

23. CUCIUREANU, A., KIM, L., LEHR, C.B., ENE, C., Rev. Chim., 68, (8), 2017, 1695.

24. BORRA C.R., MERMANS J., BLANPAIN B., PONTIKES Y., BINNEMANS K., VAN GERVEN T., Miner. Eng, 92, 2016, p.151.

25. XUAN CHIEN N., NGOC KHAI P., DUC HIEN T., NGUYEN D., CONG BOT D.,VAN TRUNG T., THI CUC N., HONG MINH L., VIET THUC N., THI NGAN B. AND VAN THUAN D., VAECAR 06-31, 2006, p.222.

26. ***ISO/CEI: Guide 98-3/2008. Uncertainty of Measurement. Part 3. Guide to the Expression of Uncertainty in Measurement (GUM: 1995).

27. EL-RAMADY, H. R., VDM-Verlag Dr. Mueller, Germany, 2010, ISBN-No. 978-3-639-23680-4. 28.***ORDER no. 756 / 1997 for the approval of the Regulation regarding the assessment of environmental pollution, p.10, in Romanian.

Manuscript received: 13.01 .2020 